\title{
Generation of mesenchymal stem cell from human umbilical cord tissue using a combination enzymatic and mechanical disassociation method.
}

\begin{abstract}
MSCs (mesenchymal stem cells) promise a great potential for regenerative medicine due to their unique properties of self-renewal, high plasticity, modulation of immune response and the flexibility for genetic modification. Therefore, the increasing demand for cellular therapy necessitates a larger-scale production of MSC; however, the technical and ethical issues had put a halt on it. To date, studies have shown that MSC could be derived from human UC (umbilical cord), which is once considered as clinical waste. We have compared the two conventional methods which are classic enzymatic digestion and explant method with our newly tailored enzymatic-mechanical disassociation method to generate UC-MSC. The generated UC-MSCs from the methods above were characterized based on their immunophenotyping, early embryonic transcription factors expression and mesodermal differentiation ability. Our results show that enzymatic-mechanical disassociation method increase the initial nucleated cell yield greatly (approximately 160-fold) and maximized the successful rate of UC-MSC generation. Enzymatic-mechanical disassociation-derived UCMSC exhibited fibroblastic morphology and surface markers expression of CD105, CD73, CD29, CD90 and MHC class I. Furthermore, these cells constitutively express early embryonic transcription factors (Nanog, Oct-4, Sox-2 and Rex-1), as confirmed by RT-PCR, indicating their multipotency and high self-renewal capacity. They are also capable of differentiating into osteoblasts and adipocytes when given an appropriate induction. The present study demonstrates a new and efficient approach in generating MSC from UC, hence serving as ideal alternative source of mesenchymal stem cell for clinical and research use.
\end{abstract}

Keyword: Human umbilical cord; Mesenchymal stem cell; Wharton's jelly. 